\title{
金钗石斛中联苄基衍生物化学成分研究
}

\author{
张茂生 ${ }^{a}$ 令狐浪 ${ }^{a}$ 张建永 ${ }^{a}$ 聂绪强 ${ }^{a}$ \\ 李晓飞 ${ }^{b}$ 郭大乐 ${ }^{c}$ 肖世基*,a,b \\ ( ${ }^{a}$ 遵义医科大学药学院 贵州遵义 563006) \\ ( ${ }^{b}$ 贵州省兰科药用植物繁育及高效应用工程研究中心 贵州遵义 563006) \\ ( ${ }^{c}$ 成都中医药大学药学院 成都 611137)
}

\begin{abstract}
摘要 通过硅胶和 MCI 柱色谱以及制备液相色谱等分离方法从金钗石斛中分离得到 3 个联苄基类衍生物，其中化合物 1 为外消旋的双联苄基衍生物, 进一步通过手性拆分得到一对对映异构体 $1 \mathbf{a}$ 和 $1 \mathrm{~b}$, 通过计算所得的电子圆二色谱 (ECD)谱图与实测的 ECD 谱图进行比较得出 $\mathbf{1 a}$ 和 $\mathbf{1 b}$ 的绝对构型. 经高分辨质谱和核磁共振等波谱技术确立化合物 $\mathbf{1} \sim$ 3 的结构分别为二聚体石斛素 A (1)、金钗石斛素 B (2)和金钗石斛素 C (3).
\end{abstract}

关键词 金钗石斛; 兰科; 联苄基衍生物; 金钗石斛素

\section{Bibenzyl Derivatives from Dendrobium nobile}

\author{
Zhang, Maosheng ${ }^{a} \quad{\text { Linghu, } \text { Lang }^{a} \quad \text { Zhang, Jianyong }^{a} \quad \text { Nie, Xuqiang }}^{a}$ \\ Li, Xiaofei ${ }^{b}$ Guo, Dale ${ }^{c} \quad$ Xiao, Shiji*,a,b \\ ( ${ }^{a}$ School of Pharmacy, Zunyi Medical University, Zunyi, Guizhou 563006) \\ ( ${ }^{b}$ Guizhou Provincial Research Center for the Propagation and Efficient Utilization of Medicinal Orchids, \\ Zunyi, Guizhou 563006) \\ ( ${ }^{c}$ School of Pharmacy, Chengdu University of Traditional Chinese Medicine, Chengdu 611137)
}

\begin{abstract}
By silica gel, MCI column chromatographic and preparative high performance liquid chromatography (HPLC) technologies, three new bibenzyl derivatives were isolated from the tubes of Dendrobium nobile. One racemic compound was further purified by chiral (HPLC) to obtain a pair of enantiomers $\mathbf{1 a}$ and $\mathbf{1 b}$, and the absolute configurations of the enantiomers were confirmed using electronic circular dichroism (ECD) calculations. By using spectroscopic techniques including NMR and HR-ESIMS, the structures of compounds $\mathbf{1} \sim \mathbf{3}$ were identified as didendronbiline A (1), dendronbiline B (2), and dendronbiline $\mathrm{C}(3)$.

Keywords dendrobium nobile; orchidaceae; bisbibenzyl; dendronbiline
\end{abstract}

兰科(Orchidaceae)石斛属植物金钗石斛(Dendrobium nobile Lindl.)主要分布于我国四川、云南、贵州、 台湾、湖北、广东、海南和广西等省海拔 800 1700 m 的山坡林中树上或路边岩石上. 金钗石斛是我国传统名 贵中药，被国际药用植物界称为 “药界大熊猫”，贵州 省赤水金钗石斛为国家地理标志产品. 金钗石斛以茎入 药, 味甘, 性微寒, 益胃生津, 滋阴清热. 用于阴伤津 亏、口干烦渴、食少干呕、病后虚热和目暗不明 ${ }^{[1]}$. 研 究表明石斛属植物具有抗肿瘤、增强机体免疫能力、抗
氧化、抗炎、护肝及降血糖等作用 ${ }^{[2,3]}$, 其主要含有生物 碱、芳香类和倍半萜类化合物 ${ }^{[4,5]}$. 石斛属植物所含化学 成分复杂多样，不同种石斛所含化学成分不尽相同，同 种不同产地的石斛化学成分含量也有差异. 为了进一步 阐明黔产金钗石斛的化学成分 ${ }^{[6,7]}$, 为贵州省道地药材 的利用奠定基础, 对采自遵义赤水市的金钗石斛进行了 深入的研究, 从其乙醇提取物的非生物碱部位中分离得 到 3 个新的联苄类衍生物(图 1), 通过波谱技术鉴定了它 们的结构, 其中化合物 $\mathbf{1}$ 为外消旋的双联苄基衍生物,

\footnotetext{
* Corresponding author. E-mail: xiaoshiji84@163.com

Received March 20, 2019; revised April 29, 2019; published online July 2, 2019.

Project supported by the National Natural Science Foundation of China (No. 31560102), and the Key Technologies R\&D Program of Guizhou Province (No. QKH [2015]6010-2).

国家自然科学基金(No. 31560102)、贵州省科技重大专项子项目(No. 黔科合重大专项字[2015]6010-2)资助项目.
} 

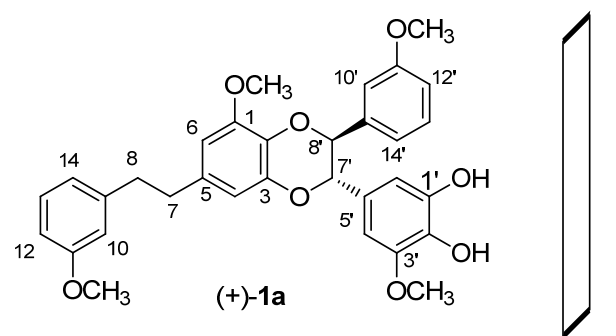<smiles>COc1cccc(CCc2cc(OC)c3c(c2)O[C@H](c2cc(O)c(O)c(OC)c2)[C@H](c2cccc(OC)c2)O3)c1</smiles><smiles>COc1cc([C+]2Oc3cc(CCc4cccc(O)c4)cc(OC)c3O[C@@H](CO)C2C)cc(O)c1O</smiles><smiles>COc1cccc(CCc2cc(OC)c3c(c2)OC(CO)C(c2cc(OC)c(O)c(OC)c2)O3)c1</smiles>

图 1 化合物 1 $\sim 3$ 的结构

Figure 1 Structures of compounds $1 \sim 3$

进一步通过手性拆分得到一对对映异构体 $1 \mathrm{a}$ 和 $1 \mathrm{~b}$, 通 过计算所得的 ECD 图谱与实测的 ECD 图谱进行比较得 到 $1 \mathrm{a}$ 和 $1 \mathrm{~b}$ 的绝对构型. 化合物 $1 \sim 3$ 的结构分别命名为 二聚体石斛素 A (1), 金钗石斛素 B (2)和金钗石斛素 C (3).

\section{1 结果与讨论}

\section{1 化合物 1 的结构鉴定}

灰白色粉末, HR-ESIMS 给出化合物的分子式为 $\mathrm{C}_{32} \mathrm{H}_{32} \mathrm{O}_{8}$ (测量值 $m / z 545.2169[\mathrm{M}+\mathrm{H}]^{+}$, 计算值为

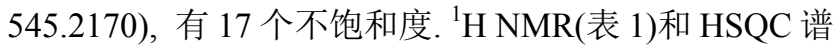
图显示有 2 个亚甲基 $\left[\delta_{\mathrm{H}} 2.82 \sim 2.93(\mathrm{~m}, 2 \mathrm{H} \times 2)\right] ; 4$ 个甲 氧基 $\left[\delta_{\mathrm{H}} 3.67,3.71,3.81,3.84\right.$ (各 $\left.\left.3 \mathrm{H}, \mathrm{s}\right)\right] ; 2$ 个连氧的次甲 基 $\left[\delta_{\mathrm{H}} 4.73(\mathrm{~d}, J=8.0 \mathrm{~Hz}, 1 \mathrm{H}), 4.82(\mathrm{~d}, J=8.0 \mathrm{~Hz}, 1 \mathrm{H})\right]$; 还有 12 个芳香质子, 包括 2 个 1,3 -二取代苯环 $\left[\delta_{\mathrm{H}} 7.22\right.$ (br t, $J=8.0 \mathrm{~Hz}, 1 \mathrm{H}$ ), 6.81 (br d, $J=8.0 \mathrm{~Hz}, 1 \mathrm{H}), 6.75$ (br d, $J=8.0 \mathrm{~Hz}, 1 \mathrm{H}$ )和 $\delta_{\mathrm{H}} 7.15$ (br. t, $J=8.0 \mathrm{~Hz}, 1 \mathrm{H}$ ), 6.78 $(1 \mathrm{H}$, br. d, $J=8.0 \mathrm{~Hz}), 6.66$ (br. d, $J=8.0 \mathrm{~Hz}, 1 \mathrm{H})$ ], 以及 6 个间位质子信号 $\left[\delta_{\mathrm{H}} 6.09,6.37,6.46,6.57,6.66,6.77\right.$ (各 $1 \mathrm{H}, \mathrm{br} . \mathrm{s})] \cdot{ }^{13} \mathrm{C}$ NMR 数据(表 1) 结合 HSQC 谱显示高场有 2 个亚甲基碳信号 $\left[\delta_{\mathrm{C}} 37.6,38.0\right] ; 4$ 个甲氧基碳信号 $\left[\delta_{\mathrm{C}}\right.$ $55.2,55.3,56.1,56.2] ; 2$ 个连氧的次甲基碳信号 $\left[\delta_{\mathrm{C}} 80.5\right.$, 80.5]; 24 个芳香区碳信号 $\left[\delta_{\mathrm{C}} 103.0 \sim 159.6\right]$. 基于以上信 息, 推测化合物 1 是一个双联苄基衍生物.

HMBC 相关谱图中 H-4 和 C-2, C-3, C-5, C-6, C-7 以及 H-8 和 C-5, C-7, C-9, C-10, C-14 有相关(图 2), 确定 一个联苄单元; HMBC 相关, 谱中 H-7'和 C-4', C-5', C-6', C-8'以及 H-8'和 C-7', C-9', C-10', C-14'有相关确定另外 一个联苄单元. HMBC 相关谱中, H-6 和 C-1, C-2, C-4 有 相关, $\delta_{\mathrm{H}} 3.79\left(1-\mathrm{OCH}_{3}\right)$ 和 C-1 有相关, 确定一个甲氧基 在 C-1 上. 同理从 $\mathrm{HMBC}$ 相关谱上确定另外的 3 个甲氧

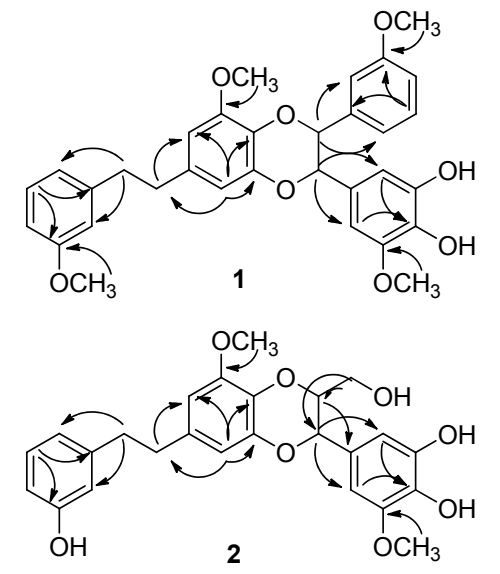

图 2 化合物 1 和 $\mathbf{2}$ 的主要的 HMBC ( $\frown$ )相关

Figure 2 Key HMBC $(\frown$ ) correlations of compounds 1 and 2

基分别连在 C-11', C-1'和 C-11'上. 普通 HMBC 谱中未发 现两个联苄单元之间有相关信号 ${ }^{[8,9]}$. 通过查找文献发 现两个联苄单元有可能通过联苯方式 ${ }^{[10,11]}$ 或者是 1,4 -二 氧六环方式连接 ${ }^{[12,13]}$. 通过联苯方式连接的化合物 dendronophenol $\mathrm{A}^{[10]}$ 和化合物 $\mathbf{1}$ 的核磁数据基本一致, 很有可能文献结构解析有错误, 因为假如是联苯形式连 接, 连接 2 个苯环的碳原子会因为旁边羟基和甲氧基的 屏蔽作用向高场移动, 其化学位移值将远小于 $130^{[14,15]}$, 其碳谱数据的模拟值分别为 $\delta 108.6$ 和 110.3 跟实际测得 值 $\delta 131.7$ 和 132.5 相差太大, 而且联苯形式往往会有阻 转异构现象存在 ${ }^{[16]}$. 当两个联苄单元通过 $1,4-$ 二氧六环 方式连接时, C-2 (131.7)和 C-2' (132.5)化学位移值和文 献中联茮基类衍生物的相近 ${ }^{[6]}$, 其碳谱数据的模拟值分 别为 $\delta 133.3$ 和 135.6, 和实际测得相差较小, 于是我们 推测两个联芐单元是通过 1,4-二氧六环方式连接的. 进 一步通过对比文献发现化合物 $\mathbf{1}$ 和 dendrocandin I 结构 非常相似 ${ }^{[13]}$, 差别在于 dendrocandin I 的对位取代联苄 变成了化合物 1 的间位取代. 通过分析 H-7'和 H-8'之间 
表 1 化合物 $1 \sim 3$ 的 ${ }^{1} \mathrm{H} \mathrm{NMR}$ 和 ${ }^{13} \mathrm{C} \mathrm{NMR}$ 数据(400/100 MHz)

Table $1{ }^{1} \mathrm{H}$ NMR and ${ }^{13} \mathrm{C}$ NMR $(400 / 100 \mathrm{MHz})$ data of compounds $\mathbf{1} \sim \mathbf{3}$

\begin{tabular}{|c|c|c|c|c|c|c|}
\hline \multirow{2}{*}{ No. } & \multicolumn{2}{|l|}{$\mathbf{1}^{a}$} & \multicolumn{2}{|c|}{$2^{b}$} & \multicolumn{2}{|c|}{$3^{a}$} \\
\hline & $\delta_{\mathrm{H}}(J$ in $\mathrm{Hz})$ & $\delta_{\mathrm{C}}$ & $\delta_{\mathrm{H}}(J$ in $\mathrm{Hz})$ & $\delta_{\mathrm{C}}$ & $\delta_{\mathrm{H}}(J$ in $\mathrm{Hz})$ & $\delta_{\mathrm{C}}$ \\
\hline 1 & & 148.7 & & 149.8 & & 148.5 \\
\hline 2 & & 131.7 & & 132.6 & & 131.2 \\
\hline 3 & & 143.6 & & 145.2 & & 144.3 \\
\hline 4 & 6.57 (br s) & 109.3 & $6.43(\mathrm{~d}, 1.8)$ & 110.2 & $6.52(\mathrm{~d}, 1.8)$ & 109.7 \\
\hline 5 & & 134.3 & & 134.7 & & 134.5 \\
\hline 6 & 6.37 (br s) & 104.8 & $6.46(\mathrm{~d}, 1.8)$ & 106.0 & $6.34(\mathrm{~d}, 1.8)$ & 104.9 \\
\hline 7 & $2.82 \sim 2.93(\mathrm{~m})$ & 37.6 & $2.76 \sim 2.85(\mathrm{~m})$ & 38.3 & $2.81 \sim 2.91(\mathrm{~m})$ & 37.7 \\
\hline 8 & $2.82 \sim 2.93(\mathrm{~m})$ & 38.0 & $2.76 \sim 2.85(\mathrm{~m})$ & 38.6 & $2.81 \sim 2.91(\mathrm{~m})$ & 38.1 \\
\hline 9 & & 143.4 & & 144.5 & & 143.4 \\
\hline 10 & 6.77 (br s) & 114.2 & $6.72($ br s) & 116.3 & 6.74 (br s) & 114.5 \\
\hline 11 & & 159.4 & & 158.3 & & 159.8 \\
\hline 12 & $6.75(\mathrm{br} \mathrm{d}, 8.0)$ & 111.2 & $6.65(\mathrm{brd}, 7.7)$ & 113.7 & $6.75(\mathrm{br} \mathrm{d}, 7.6)$ & 111.3 \\
\hline 13 & $7.22(\mathrm{br} \mathrm{t}, 8.0)$ & 129.2 & 7.09 (br t, 7.7) & 130.1 & $7.21(\mathrm{brt}, 7.6)$ & 129.5 \\
\hline 14 & $6.81(\mathrm{br} \mathrm{d}, 8.0)$ & 120.9 & $6.71(\mathrm{brd}, 7.7)$ & 120.5 & $6.79(\mathrm{br} \mathrm{d}, 7.6)$ & 121.0 \\
\hline $1^{\prime}$ & & 146.5 & & 146.3 & & 147.5 \\
\hline $2^{\prime}$ & & 132.5 & & 135.1 & & 135.4 \\
\hline $3^{\prime}$ & & 144.0 & & 149.0 & & 147.5 \\
\hline $4^{\prime}$ & 6.09 (br s) & 103.0 & $6.67(\mathrm{~d}, 2.0)$ & 103.8 & $6.68(\mathrm{~s})$ & 104.3 \\
\hline $5^{\prime}$ & & 127.8 & & 128.9 & & 127.5 \\
\hline $6^{\prime}$ & 6.46 (br s) & 108.4 & $6.65(\mathrm{~d}, 2.0)$ & 109.4 & $6.68(\mathrm{~s})$ & 104.3 \\
\hline $7^{\prime}$ & $4.73(\mathrm{~d}, 8.0)$ & 80.5 & $4.88(\mathrm{~d}, 7.9)$ & 77.2 & $4.96(\mathrm{~d}, 8.2)$ & 76.6 \\
\hline $8^{\prime}$ & $4.82(\mathrm{~d}, 8.0)$ & 80.5 & $3.95 \sim 4.02(\mathrm{~m})$ & 79.3 & $3.97 \sim 3.99(\mathrm{~m})$ & 78.5 \\
\hline $9^{\prime}$ & & 137.9 & $\begin{array}{l}3.49 \sim 3.55(\mathrm{~m}), \\
3.75 \sim 3.77(\mathrm{~m})\end{array}$ & 61.9 & $\begin{array}{l}3.56(\mathrm{dd}, 12.6,5.6) \\
3.90(\mathrm{dd}, 12.6,2.7)\end{array}$ & 61.7 \\
\hline $10^{\prime}$ & $6.66(\mathrm{br} \mathrm{s})$ & 112.9 & & & & \\
\hline $11^{\prime}$ & & 159.6 & & & & \\
\hline $12^{\prime}$ & $6.78(\mathrm{br} \mathrm{d}, 8.0)$ & 114.2 & & & & \\
\hline $13^{\prime}$ & $7.15($ br t, 8.0) & 129.3 & & & & \\
\hline $14^{\prime}$ & $6.66(\mathrm{br} \mathrm{d}, 8.0)$ & 120.1 & & & & \\
\hline $1-\mathrm{OCH}_{3}$ & $3.84(\mathrm{~s})$ & 56.1 & $3.79(\mathrm{~s})$ & 56.3 & $3.80(\mathrm{~s})$ & 56.2 \\
\hline $11-\mathrm{OCH}_{3}$ & $3.71(\mathrm{~s})$ & 55.3 & & & $3.85(\mathrm{~s})$ & 55.3 \\
\hline $1^{\prime}-\mathrm{OCH}_{3}$ & $3.81(\mathrm{~s})$ & 56.2 & & & $3.92(\mathrm{~s})$ & 56.6 \\
\hline 3'- $\mathrm{OCH}_{3}$ & & & $3.84(\mathrm{~s})$ & 56.6 & $3.92(\mathrm{~s})$ & 56.6 \\
\hline $11^{\prime}-\mathrm{OCH}_{3}$ & $3.67 \mathrm{~s}$ & 55.2 & & & & \\
\hline
\end{tabular}

${ }^{a}$ In $\mathrm{CDCl}_{3} ;{ }^{b}$ in $\mathrm{CD}_{3} \mathrm{COCD}_{3}$.

的偶合常数 $(J=8.0 \mathrm{~Hz})$, 推测出其相对构型为反式 ${ }^{[9]}$. 因为化合物的旋光值接近零, 我们推测化合物 1 为外消 旋体 ${ }^{[17,18]}$. 进一步通过手性 HPLC 分离将化合物 $\mathbf{1}$ 拆分 为一对对映体 $(+)-1 \mathrm{a}$ 和(一)-1b. 通过比较实验测得的 和计算得到的 ECD 谱(图 3), $\mathbf{1 a}$ 和 $\mathbf{1 b}$ 的绝对构型得到了 确立. 综上所述, 化合物 $1 \mathbf{a}$ 和 $1 \mathbf{b}$ 鉴定为: $\left(7^{\prime} S, 8^{\prime} S\right)$-二聚 体石斛素 $\mathrm{A}(\mathbf{1 a})$ 和 $\left(7^{\prime} R, 8^{\prime} R\right)-$ 二聚体石斛素 $\mathrm{A}(\mathbf{1 b})$.

\section{2 化合物 2 的结构鉴定}

灰白色粉末, HR-ESIMS 给出化合物的分子式为 $\mathrm{C}_{25} \mathrm{H}_{26} \mathrm{O}_{8}$ (测量值 $m / z 455.1700[\mathrm{M}+\mathrm{H}]^{+}$, 计算值为 455.1700), 有 13 个不饱和度. ${ }^{1} \mathrm{H} \operatorname{NMR}($ 表 1)和 HSQC 谱 图显示有 2 个亚甲基 $\left[\delta_{\mathrm{H}} 2.76 \sim 2.85(\mathrm{~m}, 2 \mathrm{H} \times 2)\right] ; 2$ 个甲 氧基 $\left[\delta_{\mathrm{H}} 3.79,3.84\right.$ (各 $\left.\left.3 \mathrm{H}, \mathrm{s}\right)\right] ; 2$ 个连氧的次甲基 $\left[\delta_{\mathrm{H}}\right.$
$3.95 \sim 4.02(1 \mathrm{H}, \mathrm{m}), 4.88(\mathrm{~d}, J=7.9 \mathrm{~Hz}, 1 \mathrm{H})] ; 1$ 个连氧的 亚甲基 $\left[\delta_{\mathrm{H}} 3.49 \sim 3.55,3.75 \sim 3.77\right.$ (各 $1 \mathrm{H}, \mathrm{m}$ ) ]; 还有 8 个芳 香质子信号[包括 1 个 1,3 -二取代苯环 $\delta_{\mathrm{H}} 7.09(\mathrm{t}, J=7.7$ $\mathrm{Hz}, 1 \mathrm{H}), 6.71$ (d, $J=7.7 \mathrm{~Hz}, 1 \mathrm{H}), 6.65$ (d, $J=7.7 \mathrm{~Hz}, 1 \mathrm{H})$, $6.72(\mathrm{br} \mathrm{s}, 1 \mathrm{H})]$, 以及 4 个间位质子信号 $\left[\delta_{\mathrm{H}} 6.43,6.46\right.$ (各 $1 \mathrm{H}, \mathrm{d}, J=1.8 \mathrm{~Hz}$ ); 6.65, 6.67 (各 $1 \mathrm{H}, \mathrm{d}, J=2.0 \mathrm{~Hz}$ )]. ${ }^{13} \mathrm{C}$ NMR 数据(表 2)结合 HSQC 谱显示高场有 2 个亚甲基碳 信号 $\left(\delta_{\mathrm{C}} 38.3,38.6\right) ; 2$ 个甲氧基碳信号 $\left(\delta_{\mathrm{C}} 56.3,56.6\right) ; 2$ 个连氧的次甲基碳信号 $\left(\delta_{\mathrm{C}} 77.2,79.3\right) ; 1$ 个连氧的亚甲 基碳信号 $\left(\delta_{\mathrm{C}} 61.9\right) ; 18$ 个芳香区碳信号 $\left(\delta_{\mathrm{C}} 103.8 \sim 158.3\right)$. 基于以上信息，推测化合物 2 是一个联茮基衍生物. 通 过对比发现, 化合物 2 比化合物 1 少了一个苯环和 2 个 甲氧基，多了一个羟甲基. 进一步通过查找文献发现化 


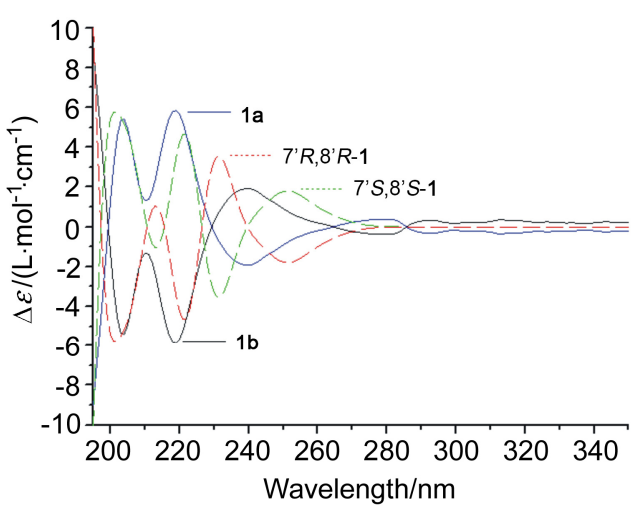

图 3 在甲醇中化合物 $\mathbf{1}$ 的实验 $\mathrm{ECD}$ 谱图和 $7^{\prime} S, 8^{\prime} S-1$ 及 $7^{\prime} R$, $8^{\prime} R-1$ 计算 $\mathrm{ECD}$ 谱图

Figure 3 Experimental ECD spectra of $\mathbf{1}$ and calculated ECD spectra of $7^{\prime} S, 8^{\prime} S-1$ and $7^{\prime} R, 8^{\prime} R-1$ in $\mathrm{MeOH}$

UV correction $3 \mathrm{~nm}$, band width $0.20 \mathrm{eV}$

合物 2 和 dendrocandin $O$ 非常相似 ${ }^{[12]}$, dendrocandin $O$ 上 联茮基的对位取代变成了间位取代. 通过分析 H-7'和 H-8'之间的偶合常数 $(J=7.9 \mathrm{~Hz})$, 推测出其相对构型也 为反式 ${ }^{[9]}$, 通过 NOESY 谱中, H-7'和 H-9'有相关, H-8 '和 H-4', H-6'有相关进一步得到确证. 其旋光值为负, 以及 和化合物 1 的 ECD 谱比较, $220 \mathrm{~nm}$ 处有正的 Cotton 效 应, 得出 $7^{\prime}$ 和 $8^{\prime}$ 位的绝对构型为 $\left(7^{\prime} R, 8^{\prime} R\right)$, 化合物 2 命名 为金钗石斛素 B.

\section{3 化合物 3 的结构鉴定}

灰白色粉末, HR-ESIMS 给出化合物的分子式为 $\mathrm{C}_{27} \mathrm{H}_{30} \mathrm{O}_{8}$ (测量值 $m / z$ 483.2016 $[\mathrm{M}+\mathrm{H}]^{+}$, 计算值为 483.2013), 有 13 个不饱和度. ${ }^{1} \mathrm{H}$ NMR, ${ }^{13} \mathrm{C}$ NMR 数据 (表 1)和 HSQC 谱图显示有 2 个亚甲基, 4 个甲氧基, 2 个 连氧的次甲基, 1 个连氧的亚甲基, 1 个 1,3 -二取代苯环, 2 个 1,3,4,5-四取代苯环. 通过对比发现, 化合物 3 比化 合物 2 多了 2 个甲氧基. 由 ${ }^{1} \mathrm{H}$ NMR 谱中 $\delta_{\mathrm{H}} 6.68(2 \mathrm{H})$, $3.92(6 \mathrm{H})$ 推测一个苯环有对称性, 结合分子式推测有 1 个局部对称的 $1^{\prime}, 3^{\prime}$-二甲氧基苯基. 通过 $\mathrm{HMBC}$ 谱 $11-\mathrm{OCH}_{3}\left(\delta_{\mathrm{H}} 3.85\right)$ 和 C-11 $\left(\delta_{\mathrm{C}} 159.8\right)$ 有相关, 得出另外一 个甲氧基处在 C-11 位上. 由 $\mathrm{H}-7$ '和 $\mathrm{H}-8^{\prime}$ 之间的偶合常数 $(J=8.2 \mathrm{~Hz})$, 进一步推测出其相对构型也为反式 ${ }^{[9]}$, 但 其旋光值为零, ECD 谱谱线平直, 推测为外消旋体混合 物, 由于化合物量少, 没有进行手性拆分. 化合物 3 命 名为金钗石斛素 C.

\section{2 实验部分}

\section{1 仪器与试剂}

质谱用 Thermo Scientific LTQ Orbitrap XL 型质谱 仪测定; 核磁共振用 Agilent DD2 400-MR 型核磁共振 仪和 Bruker BiOTOF $\mathrm{Q}$ 型核磁共振仪测定, TMS 为内标;
IR 用 FTIR-850 傅里叶变换红外光谱仪测定, $\mathrm{KBr}$ 压片; 日本 Mitsubishi 公司 MCI 树脂; 德国 Merck 公司 Sephadex LH-20 凝胶; 薄层色谱硅胶 $\mathrm{GF}_{254}$ 和柱色谱硅 胶(300 400 目)均为青岛海洋化工厂生产; 半制备高效 液相色谱仪用北京创新通恒 LC3000 型色谱仪, HPLC 柱 用 $\mathrm{YMC} \mathrm{C}_{18}, 5 \mu \mathrm{m}, 10 \mathrm{~mm} \times 250 \mathrm{~mm}$; Daicel Chiralpak $\mathrm{AD} 10 \mu \mathrm{m}, 4.6 \mathrm{~mm} \times 250 \mathrm{~mm}$.

\section{2 植物材料}

金钗石斛茎于 2014 年 9 月购自遵义赤水, 由遵义 医科大学生药学教研室杨建文教授鉴定为金钗石斛 (Dendrobium nobile Lindl.), 样品标本(No.20141011)保 存在遵义医科大学药学院.

\section{3 提取分离}

金钗石斛(干重 $50 \mathrm{~kg}$ )粉碎后用乙醇回流提取 3 次, 减压浓缩提取液得乙醇提物 $4.8 \mathrm{~kg}$, 将其分散于 $2.0 \%$ 的盐酸水溶液中, 减压过滤得酸水不溶物. 该酸水不溶 物用乙醇溶解后拌入 $100 \sim 200$ 目硅胶, 挥干溶剂后先 用石油醚提取 (3 L), 脱脂后以石油醚-乙酸乙酯 $(V$ : $V=1: 1,3 \mathrm{~L})$ 和乙酸乙酯 $(3 \mathrm{~L})$ 分别提取三次, 减压浓缩 得石油醚-乙酸乙酯部位浸膏 $350 \mathrm{~g}$. 该部位浸膏经硅胶 柱色谱(石油醚一乙酸乙酯梯度洗脱, $V: V=100$ : $1 \rightarrow 1: 100)$ 分为 8 个部分 Fr.1 Fr.8. 第二部分 Fr.2 经 MCI 柱色谱分离, 90\%甲醇-水洗脱，除去色素后经 ODS 柱色谱分离 30\% 90\%甲醇-水梯度洗脱，分为 4 个部分 Fr.2.1 Fr.2.4. Fr.2.4 经半制备 HPLC 分离 $[V(\mathrm{MeCN})$ : $\left.V\left(\mathrm{H}_{2} \mathrm{O}\right)=82: 18,4.0 \mathrm{~mL} / \mathrm{min}\right]$ 得到化合物 $\mathbf{1}\left(t_{R}=13.7\right.$ $\mathrm{min}, 50 \mathrm{mg}$ ). 化合物 $\mathbf{1}$ 通过手性 HPLC 柱色谱分离 $[V$ (正 己烷)：V(异丙醇 $)=65: 35,3.0 \mathrm{~mL} / \mathrm{min}$ ]得到一对对映 体化合物 1a 和 1b. Fr.6 进一步经经 MCI 柱色谱分离, 分为 4 个部分 Fr.6.1 Fr.6.4. Fr.6.2 经 Sephadex LH-20 凝胶柱色谱分离得到 6 个部分 Fr.6.2.1 Fr.6.2.6. Fr.6.2.2 经半制备 HPLC $\left[V(\mathrm{MeOH}): V\left(\mathrm{H}_{2} \mathrm{O}\right)=70: 30,3.0\right.$ $\mathrm{mL} / \mathrm{min}$ ]分离得到化合物 $2\left(t_{\mathrm{R}}=19.7 \mathrm{~min}, 15 \mathrm{mg}\right)$. Fr.6.2.3 经半制备 HPLC $\left[V(\mathrm{MeOH}): V\left(\mathrm{H}_{2} \mathrm{O}\right)=85: 15\right.$, $3.0 \mathrm{~mL} / \mathrm{min}$ ]分离得到化合物 $3\left(t_{\mathrm{R}}=13.0 \mathrm{~min}, 8 \mathrm{mg}\right)$.

二聚体石斛素 $\mathrm{A}(\mathbf{1})$ ：灰白色粉末, $[\alpha]_{\mathrm{D}}^{20}+18(c 0.10$, $\mathrm{MeOH})(\mathbf{1 a}) ;[\alpha]_{\mathrm{D}}^{20}-19$ (c 0.12, MeOH) (1b); ${ }^{1} \mathrm{H}$ NMR 和 ${ }^{13} \mathrm{C}$ NMR 数据见表 1 ; UV $(\mathrm{MeOH}) \lambda_{\max }[\log \varepsilon /(\mathrm{L} \cdot$ $\left.\mathrm{mol}^{-1} \cdot \mathrm{cm}^{-1}\right)$ ]: $273(2.08) \mathrm{nm}$; IR (KBr) v: 3432, 2937, 1602, 1514, 1454, 1317, 1219, 1117, 1049, 783, $697 \mathrm{~cm}^{-1}$; HR-ESI-MS calcd for $\mathrm{C}_{32} \mathrm{H}_{33} \mathrm{O}_{8}[\mathrm{M}+\mathrm{H}]^{+}$545.2170, found 545.2169.

金钗石斛素 B (2): 灰白色粉末, $[\alpha]_{\mathrm{D}}^{20}-16$ (c 0.18 , $\mathrm{MeOH}) ;{ }^{1} \mathrm{H}$ 和 ${ }^{13} \mathrm{C}$ NMR 数据见表 1 ; UV $(\mathrm{MeOH}) \lambda_{\text {max }}$

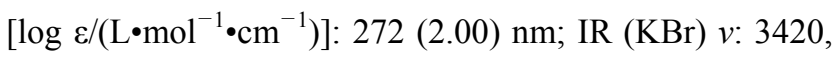


1601, 1454, 1346, 1220, 1116, 1051, $831 \mathrm{~cm}^{-1}$; HR-ESIMS calcd for $\mathrm{C}_{25} \mathrm{H}_{27} \mathrm{O}_{8}[\mathrm{M}+\mathrm{H}]^{+}$455.1700, found 455.1700 .

金钗石斛素 C (3): 灰白色粉末, $[\alpha]_{\mathrm{D}}^{20} 0$ ( 0 ( 0.10 , $\mathrm{MeOH}) ;{ }^{1} \mathrm{H}$ NMR 和 ${ }^{13} \mathrm{C} \mathrm{NMR}$ 数据见表 $1 ; \mathrm{UV}(\mathrm{MeOH})$

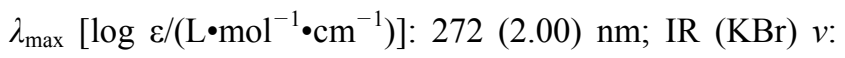
3482, 1601, 1510, 1463, 1340, 1220, 1117, 1048, 835 $\mathrm{cm}^{-1}$; HR-ESI-MS calcd for $\mathrm{C}_{27} \mathrm{H}_{31} \mathrm{O}_{8}[\mathrm{M}+\mathrm{H}]^{+}$ 483.2013, found 483.2016.

辅助材料(Supporting Information) 化合物 $\mathbf{1} \sim \mathbf{3}$ 的 ${ }^{1} \mathrm{H}$ $\mathrm{NMR},{ }^{13} \mathrm{C} \mathrm{NMR}, \mathrm{HSQC}, \mathrm{HMBC}, \mathrm{NOESY}$ 谱和 HR-ESI$\mathrm{MS}$ 谱图. 这些材料可以免费从本刊网站(http://siocjournal.cn/)上下载.

\section{References}

[1] Zhang, H. Y.; Zhang, Z. Y. The Chinese Traditional Medicine Resource Records, Science Press, Beijing, 1994 (in Chinese). (张惠源, 张志英, 中国中药资源志要, 科学出版社, 北京, 1994.)

[2] Xu, J.; Han, Q. B.; Li, S. L.; Wang, X. N.; Zhao, Z. Z.; Chen, H. B. Phytochem. Rev. 2013, 12, 341.

[3] Zhang, X. Q.; Zhao, T. M.; Liu, J.; Zhao, R. X.; Zheng, S. G.; Ze, C.; Hu, Y. D. Chin. Tradit. Herb. Drugs 2018, 49, 3174 (in Chinese).

(张雪琴, 赵庭梅, 刘静, 赵若茜, 郑世刚, 淳泽, 胡亚东, 中草 药, 2018, 49, 3174.)

[4] Yang, H.; Sung, S. H.; Kim, Y. C. J. Nat. Prod. 2007, 70, 1925.

[5] Zhou, X. M.; Zheng, C. J.; Gan, L. S.; Chen, G. Y.; Zhang, X. P.; Song, X. P.; Li, G. L.; Sun, C. G. J. Nat. Prod. 2016, 79, 1791.
[6] Xiao, S. J.; Liu, Z.; Zhang, M. S.; Chen, Y. Z.; Nie, X. Q.; Zhang, J. Y.; He, Y. Q.; Shi, J. S. Acta Pharm. Sin. 2016, 51, 1117 (in Chinese).

(肖世基, 刘珍, 张茂生, 陈永正, 聂绪强, 张建永, 何芋岐, 石 京山，药学学报, 2016, 51, 1117.)

[7] Xiao, S. J.; Qian, Y.; Zhang, L.; Tang, Y. F.; Zhu, X. M.; Zhou, H. X.; Zhao, Z. N.; Zhang, M. S.; Xu, D. L. Chin. Tradit. Herb. Drugs 2016, 47, 2972 (in Chinese).

(肖世基, 钱怡, 张良, 唐艳芬, 朱雪梅, 周惠黠, 赵忠能, 张茂 生, 徐德林, 中草药, 2016, 47, 2972.)

[8] Xiang, T.; Uno, T.; Ogino, F.; Ai, C.; Duo, J.; Sankawa, U. Chem. Pharm. Bull. 2005, 53, 1204.

[9] Lee, D.; Cuendet, M.; Vigo, J. S.; Graham, J. G.; Cabieses, F.; Fong, H. H.; Pezzuto, J. M.; Kinghorn, A. D. Org. Lett. 2001, 3, 2169.

[10] Liu, Q. F.; Chen, W. L.; Tang, J.; Zhao, W. M. Helv. Chim. Acta 2007, 90, 1745.

[11] Yang, D.; Cheng, Z. Q.; Yang, L.; Hou, B.; Yang, J.; Li, X. N.; Zi, C. T.; Dong, F. W.; Liu, Z. H.; Zhou, J.; Ding, Z. T.; Hu, J. M. J. Nat. Prod. 2018, 81, 227.

[12] Li, Y.; Wang, C. L.; Zhao, H. J.; Guo, S. X. J. Asian Nat. Prod. Res. 2014, 16, 1035.

[13] Li, Y.; Wang, C. L.; Wang, Y. J.; Wang, F. F.; Guo, S. X. Yang, J. S.; Xiao, P. G. Chem. Pharm. Bull. 2009, 57, 997.

[14] Termentzi, A.; Zervou, M.; Kokkalou, E. Food Chem. 2009, 116, 371.

[15] Jiang, S.; Wang, K.; Lou, H. Y.; Yi, P.; Zhang, N.; Zhou, M.; Song, Z. Q.; Wang, W.; Wu, M. K.; Pan, W. D. Phytochemistry 2019, 162, 216.

[16] Xiao, S. J.; He, D. H.; Fang, D. M.; Chen, F.; Ding, L. S.; Zhou, Y. Helv. Chim. Acta 2014, 97, 499.

[17] Guo, D. L.; Li, X. H.; Feng, D.; Jin, M. Y.; Cao, Y. M.; Cao, Z. X.; Gu, Y. C.; Geng, Z.; Deng, F.; Deng, Y. Molecules 2018, 23, 1709.

[18] Liu, H. S.; Zhu, G. L.; Zhao, S. G.; Fu, P.; Zhu, W. M. Chin. J. Org. Chem. 2019, 39, 507 (in Chinese). (刘海珊, 朱国良, 赵水鸽, 付鹏, 朱伟明, 有机化学, 2019, 39, 507.) 